\title{
ALCANCE NORMATIVO DEL RÉGIMEN DE PROTECCIÓN AL CONSUMIDOR DE LOS SERVICIOS DE SALUD*
}

\author{
Fecha de recepción: 5 de diciembre de 2016 \\ Fecha de evaluación: 29 de julio de 2017 \\ Fecha de aprobación: 7 de noviembre de 2017 \\ Artículo de reflexión
}

Andrea Carolina Pérez Forero**

DOI: http://dx.doi.org/10.18359/prole.3330

Forma de citación: Pérez, A. C. (2018). Alcance normativo del régimen de protección al consumidor de los servicios de salud. Revista Prolegómenos Derechos y Valores, 21, 41, 61-78. DOI: http://dx.doi.org/10.18359/prole.3330

\section{RESUMEN}

Con sustento en el rediseño que tuvo la prestación de los servicios de salud a partir de una visión económica de los mismos y las consecuencias que ello acarrea; el presente escrito expondrá si en efecto existe un régimen más garantista de los derechos que como consumidor detenta quien es usuario de los servicios de salud, a quien a falta de regulación en el estatuto general, por vía de otras alternativas, se le han salvaguardado todas sus facultades; en comparación con la regulación ofrecida para los consumidores de otro tipo de servicios. Para ello y a través de una metodología teórica de corte analítico, se identificará la afinidad y disparidad normativa, determinándose que en la actualidad coexisten regímenes que se complementan en procura del bienestar de los pacientes-consumidores.

\section{Palabras clave:}

Consumidor, servicios de salud, garantía, protección contractual.

\footnotetext{
El presente artículo es resultado de la investigación propuesta como opción de grado para la maestría en Derecho, con énfasis en Derecho Privado de la Universidad del Rosario (Bogotá, Colombia), bajo tutoría del doctor Francisco José Ternera Barrios. Cursada gracias al apoyo de la Universidad Militar Nueva Granada (Bogotá, Colombia), institución para la que se exalta el debido agradecimiento y reconocimiento por el beneficio otorgado.

** Abogada magna cum laude de la Universidad Militar Nueva Granada (Bogotá, Colombia). Especialista en Derecho Contractual y en Derecho Comercial de la Universidad del Rosario (Bogotá, Colombia). Diplomada en Docencia Universitaria, Didáctica Universitaria y Diseño y Apropiación de TIC. Docente de planta de la Facultad de Derecho de la Universidad Militar Nueva Granada. Correo electrónico: andrea.perez@unimilitar.edu.co
} 


\title{
LEGAL SCOPE OF THE HEALTH SERVICES CONSUMER PROTECTION REGIMEN
}

\begin{abstract}
SUMMARY
Based on the healthcare services redesign since an economic vision and the consequences it entails, this article will set forth whether or not there is a more protective regimen with regards to the rights held by a healthcare consumer, to whom as a result of the lack of regulation in the general statute, has been granted (through different alternatives) all the faculties in contrast to the regulation offered to consumer of a different array of services. In order to reach this purpose, through a methodologic theory of analytic style will be identified a normative affinity and mismatch, determining that currently two regimens coexist, and they complement each other seeking patients-consumers welfare.
\end{abstract}

\section{Keywords:}

Consumer, health services, warranty, contractual protection.

\section{ÂMBITO NORMATIVO DO REGIME DE PROTEÇÃO AO CONSUMIDOR DOS SERVIÇOS DE SAÚDE}

\begin{abstract}
RESUMO
Com o apoio no redesenho que teve a prestação dos serviços de saúde a partir de uma visão econômica dos mesmos e as conseqüências que isso implica; o presente documento mostrará se, de fato, existe um regime mais garantido sobre os direitos que, como um consumidor detém quem são usuários dos serviços de saúde, para quem, na ausência de regulamentação no estatuto geral, por meio de outras alternativas, tem-se salvaguardado todas suas faculdades; em comparação com o regulamento oferecido para os consumidores de outro tipo de serviços. Para este fim e através de uma metodologia teórica de forma analítica, a afinidade e a disparidade normativa serão identificadas, determinando-se que atualmente coexistem regimes que se complementam em busca do bem-estar dos pacientes-consumidores.
\end{abstract}

\section{Palavras chave:}

Consumidor, serviços de saúde, garantia, proteção contratual.

\section{Introducción}

Con la llegada de un sistema de corte empresarial e institucional para la atención en salud, a tan solo dos años de haber sido promulgada la Constitución de 1991, fue apenas natural que la ciencia del derecho encontrara un motivo nuevo para destinar su espíritu y esencia regulatoria. Esta vez, desempeñando su labor en un escenario difícil en el que la salud ingresó a la economía y comenzó a asimilar al usuario como su cliente; teniendo además que darle especial significado a la delgada línea que separa y distingue a la salud como derecho esencial de la salud como servicio de obligatorio cumplimiento a cargo del recién fundado Estado social y democrático de derecho.

En este sentido, el escenario económico que rediseñó la atención en salud, comparte ideas y ambientes relativamente comunes con aquellas 
regulaciones adyacentes que tuvieron origen en asuntos de similar naturaleza y son consideradas como relaciones de consumo por el nuevo Estatuto del Consumidor. Ambos tópicos tienen tal despliegue que han establecido dinámicas de garantía y protección contractual para cada uno de sus destinatarios, a saber: el usuario, en el campo de la salud; y el consumidor en el ámbito del comercio tradicional. Sin embargo, las particulares diferencias entre estos sujetos, hacen que el acercamiento comparativo buscado - normativamente hablando- ofrezca una significativa diversidad en su aplicación, que identifica por una parte, la fortaleza de las regulaciones en materia de salud y, por otra, la consecuente inaplicabilidad del Estatuto del Consumidor a las relaciones "usuario-prestador de servicios de salud", en muchos casos por insuficiencia o incompatibilidad de sus preceptos.

En ese orden de ideas, el presente escrito expondrá si en efecto existe un régimen más garantista de los derechos que como consumidor tiene quien es usuario de los servicios de salud, puntualmente en lo que respecta a los mecanismos de reclamación que se consagran en su favor, conforme lo previsto en las leyes 100/1993, $1122 / 2007,1438 / 2011$ y 1480/2011, decretos 1011/2006 y 1018/2007 y resolución 1441/2013; normativa que integra un régimen especial que complementa al Estatuto general y que dada su aplicación preferente ha salvaguardado todas las facultades para este sector del consumo en comparación con la regulación ofrecida para los consumidores de otro tipo de servicios; sin que ello signifique que se trata de regímenes excluyentes, pues se insiste, de lo que se trata es de la complementariedad entre la norma general y las normas especiales de protección al consumidor de los servicios de salud.

Para ello se empleará una metodología predominantemente teórica de tipo analítico, a partir de la cual, se contextualiza la temática con sustento en los antecedentes sobre la materia y se estudian las garantías y protección contractual en el sector salud, considerando este el asunto de mayor interés para el consumidor, al configurar la principal herramienta cuando en una primera instancia sus intereses no han sido satisfechos. En desarrollo de lo anterior, se examinarán uno a uno los componentes del Sistema Obligatorio de Garantía de Calidad en Salud (SOGCS), mismo que se analizará a la luz de lo previsto en el Estatuto del Consumidor; finalmente se hará mención a los mecanismos de reclamación que tiene el usuario, previa referencia del régimen de responsabilidad que se tiene para con este.

\section{A. Antecedentes}

La historia demuestra cómo la sociedad ha luchado por garantizar derechos como la salud, que ha progresado con muchos sacrificios hasta hallar lugar en el mundo globalizado que hoy apreciamos y que al parecer, se interesa por el respeto de su propia humanidad así como descarta cualquier discusión retrógrada sobre la legitimidad de los derechos ya reconocidos, limitando el debate universal de derechos humanos a sopesar las medidas necesarias para promover y extender su efectividad y realización en todas las esferas sociales.

Pero el debate ha transmutado el rumbo en muchos lugares a pesar de todo el esfuerzo hecho; la salud por ejemplo, se ha transformado, dejando de ser simplemente un derecho inherente a la persona, para pasar a plantearse desde una óptica más propia del comercio y de la economía, que en muchas ocasiones cede al interés individual sobre el general, y que para el caso colombiano, resulta un factor para evolucionar y satisfacer un mínimo de calidad que garantice los derechos de la sociedad.

En esta ocasión, la visión con la que se aborda la salud parte de una perspectiva jurídica adaptada al escenario actual, que es consecuencia del ingreso de aquella a la economía de mercado y su condición como nuevo elemento de consumo, generando que el derecho haya tenido que estar atento a direccionar esta evolución, y le acompañe de forma directa y permanente, comprendiendo las relaciones complejas y variadas que se observan entre paciente o usuario como 
el extremo frágil y prestador o proveedor del servicio de salud, como la institución profesional experta en la materia; dando como resultado la creación y promoción de garantías y estatutos para la protección de todo usuario del servicio, a través de normas propias del régimen de salud y eventualmente por medio de reglas contiguas, que por su proximidad conceptual tengan la vocación de servir de aval adicional para el usuario.

El escenario es jurídico entonces, pero matizado con las relaciones de consumo en la economía, pues el derecho aplicado a la salud se ha desarrollado para abarcar el nuevo entorno donde esta se situó y que, se acerca mucho al universo de actividades que reciben un tratamiento jurídico especial, conocido como la defensa de los consumidores. Así, la práctica médica y las relaciones de consumo tradicionales convergen en el llamado destinatario, usuario o consumidor final de un servicio o producto y que, el Estatuto del Consumidor-ley 1480/2011-, regula de manera paralela a la normatividad del sector salud, siendo posible que ambos sistemas de protección puedan llegar a ofrecerse entre sí mecanismos para avanzar en sus objetivos de protección y regulación o también reflejar falencias o vacíos en cada uno, como se verá después.

Sirve aclarar desde ya, que el Sistema General de Seguridad Social en Salud (SGSSS) del que más adelante se expondrá lo propio en relación con el tema de investigación; no es el único sistema de salud que opera en Colombia tal y como se indicó tangencialmente al inicio del presente artículo, se pueden encontrar varios regímenes especiales con garantías propias y en especial adquiridas e irrenunciables para sus titulares que no pueden pasarse por alto en este estudio; no obstante, respecto a ellos se hace a continuación una breve referencia en la que no se profundizará, dado que estos condensan otra serie de normas especiales de necesario análisis en otro escrito.

Estos sistemas tienen como objetivo principal ofrecer los servicios en salud a un usuario cualificado o especial, que es determinado por el vínculo laboral o asimilable que este tiene con una institución interesada en garantizar la prestación directa de atención a sus empleados y a sus familias. En cualquier caso, así como el control de la Superintendencia Nacional de Salud tiene la vocación de introducirse a cada uno de estos regímenes, se debe entender que los mínimos exigidos por el SGSSS y las directrices mínimas extensibles del SOGCS, tienen que verse aplicados en cada uno de ellos, por lo que lo verdaderamente importante de observar son sus aportes adicionales.

Dentro de los regímenes especiales está el sistema de salud de las Fuerzas Militares creado en el año 1994; el sistema de salud del magisterio, que inició operaciones en 1989 para ofrecer servicios médico-asistenciales al personal docente y sus familias, mismo que opera el Fondo Nacional de Prestaciones Sociales del Magisterio; el sistema de salud de Ecopetrol como sistema excepcional y el sistema especial de salud de las universidades (Supersalud, 2011).

Cada uno de ellos enfoca su propio marco delimitado e invierte en el diseño de políticas propias a favor del usuario cualificado que atiende, por lo mismo, el examen de sus garantías particulares puede abordarse con mayor profundidad en un nuevo espacio, pues es muy interesante equiparar garantías y protección contractual entre sistemas de regulación afines con la misma esencia y materia prima; de hecho se pueden señalar al respecto, algunos de los atributos especiales que tan solo uno de estos sistemas ofrece con una perspectiva amplia de garantía y protección contractual en salud.

El ejemplo lo enseña el sistema excepcional de salud de Ecopetrol, reconocido por su generosidad en ciertos aspectos aún no extendidos a otros sectores de la prestación nacional, donde vale la pena mencionar que algunas limitaciones como las famosas preexistencias, se abolieron en su totalidad al momento de atender el tratamiento de enfermedades, afecciones o malformaciones previas al ingreso del usuario al sistema, asimismo los periodos de carencia de la atención y 
los límites que existen en otros sistemas frente al costo máximo cubierto de las prestaciones, fueron también suprimidos.

\section{B. Ingreso de la salud a la economía y al sector empresarial}

E origen de este entorno en Colombia puede entenderse con la ley 100/1993, que estableció hace ya dos décadas cambios significativos en materia de salud, empezando con la descentralización en la prestación del servicio, dando como resultado que los departamentos y municipios dejaran de ser los únicos que ofrecían servicios de salud de forma sistematizada, tal como había sido hasta ese entonces, abriendo una puerta para que el sector privado participara en la prestación de este servicio con la aquiescencia de la ley.

Al mismo tiempo, la prestación del servicio recibió un cambio sustancial al buscar su institucionalización y estructurarse según los principios de todo servicio público esencial, tales como eficiencia, celeridad, universalidad, igualdad y solidaridad; a la vez que su operación fue desplegándose en función de premisas como la productividad empresarial, la reorganización administrativa y el mercadeo de servicios del sector público y del sector privado de la salud (Díaz, citado en Guzmán y Redondo, 1998). Se generó así en la prestación del servicio, un ambiente mixto de intereses encontrados, donde una actividad que procura la satisfacción de un derecho humano esencial, ejerce sus funciones por medio de promotores y prestadores con un enfoque empresarial y con ánimo de lucro en la mayoría de los casos; quienes esperan lógicamente tener prosperidad y sostenibilidad económica. Todo lo que al final significa que los pacientes sean vistos, incluso por el Estado, como clientela, abandonando la mirada histórica del vínculo paciente-médico por la de proveedor-cliente, así como sucede en los demás sectores del comercio tradicional.

Este escenario exigió mantener un equilibrio entre los actores de la relación consolidando un sistema robusto de garantía y protección jurídica a través del cual, se pueda salvaguardar el acceso y la prestación de un servicio diligente y seguro a favor del usuario, atendiendo su carácter esencial y obligatorio e, igualmente, comprendiendo que el desconocimiento frente al servicio especializado que recibe el usuario, lo coloca en una posición vulnerable ante el otro extremo, donde se encuentra con un profesional dotado de fortaleza institucional y financiera, conocimiento científico y manejo pleno de la información de sus pacientes, que lo posicionan sobre una especie de pedestal al que con dificultad puede acceder el usuario si no cuenta con mecanismos que equilibren la situación.

Reconocer esa diferencia entre las partes, es lo que da cuenta de la protección sustancial en un contexto de Estado social que es plenamente distinguible de la concepción liberal basada en una relación en igualdad de condiciones y absoluta libertad de negociación entre consumidor y productor o distribuidor de bienes, o prestador de servicios, la cual es una situación ficta en la gran mayoría de los casos en que dicha relación se presenta (Corte Constitucional de Colombia, 2014).

Ante las circunstancias y el cambio que trajo consigo la ley 100/1993, se estudian las garantías y la protección aplicadas al servicio de salud, en una aproximación a la protección general que ofrece el Estatuto del Consumidor. Pero esta tarea precisa antes que todo, comprender que la prestación de los servicios públicos de salud ha tenido un escenario normativo propio con gran riqueza conceptual y normativa, muy amplia y a la vez específica originada en el derecho irrenunciable a la seguridad social y la salud así como en la compleja práctica y disciplina médica; mientras que las normas generales del Estatuto del Consumidor, abarcan de forma menos detallada la garantía y protección contractual y extracontractual en el mercado y el consumo de bienes, productos o servicios distintos a la salud, de acuerdo con reglas mucho menos limitadas y más bien orientadas por la libre competencia y el ejercicio de la autonomía de la voluntad privada.

Sin embargo, el espacio donde interactúan los prestadores del servicio de salud y los usuarios finales, presenta coincidencias que lo asemejan 
conceptualmente a la cadena de producción (Villalba, 2012) evocada por el Estatuto del Consumidor; ya que se trata de un contexto donde podemos hallar una fuente muy extensa de actores que de uno u otro modo intervienen en el aseguramiento y la prestación idónea del servicio de salud, cuya responsabilidad frente al usuario puede configurarse en la misma medida o incluso de forma más vinculante a la que tendría el productor y el proveedor ante el consumidor de otros bienes y servicios.

Así las cosas, ambos son espacios relativamente comunes de actividad en sentido general, donde participan tanto los destinatarios de un servicio como sus prestadores o proveedores, abriendo lugar para identificar comparativamente las similitudes o divergencias entre el marco jurídico que regula el servicio de salud y el propio Estatuto del Consumidor; y para desarrollar conocimiento en aquella comparación para aportar al mejoramiento de sus regulaciones como fuentes destinadas a proteger y hacer efectivos los derechos que tanto promocionan.

No obstante, debe acotarse que hay algunas posturas internacionales que todavía se oponen a equiparar al usuario del servicio de salud con el consumidor o usuario común, todo desde una perspectiva humanista, según la cual, la comparación del médico y la institución clínica con el proveedor del mercado común de servicios, ignora la naturaleza especial de este servicio y su nexo con la vida, así como la especificidad de una disciplina históricamente conocida y respetada, llegando a la llamada "banalización" de la medicina y la ciencia de la salud (Lamboley, 2004).

Ahora bien, el interés real no debe perderse de vista, pues el usuario de la salud y el de muchos otros servicios, están en una situación similar de desventaja respecto al proveedor, por lo que la posibilidad de ampliar la cobertura de un sistema que es más garantista con el usuario sea cual sea de los dos, resulta de mayor relevancia jurídica y objetiva, quedando por determinar cuál sistema aporta más al otro desde el enfoque de garantías y protección.

\section{Las garantías y protección contractual en la atención en salud}

Pues bien, el servicio de salud en Colombia, incluidos los regímenes especiales que lo conforman, puede entenderse como un gran conjunto de procesos, actores y partícipes que actúan de manera vigilada y permanente en el aseguramiento en salud y la protección de sus usuarios que precisan un servicio seguro y respetuoso de los derechos que les corresponden (Ministerio de Salud y Protección Social, 2007). En este sentido, su prestación debe comprender el marco legal y el profesional, así como la debida atención del usuario en los trámites administrativos y en la fase médico-asistencial, que garanticen un esquema óptimo de protección, calidad, seguridad $e$ información en todos los procedimientos y etapas del servicio, incluida la promoción y prevención, el diagnóstico clínico y el tratamiento preventivo o de rehabilitación; dada la responsabilidad que significa la prestación de un servicio público esencial que efectiviza un derecho humano vital de la población.

El marco general imperante en Colombia como guía en la prestación del servicio de salud es conocido como SGSSS, que se conforma por la Constitución Política de Colombia, lo define ley estatutaria 100/1993 y lo reglamentan los amplios preceptos legales que el Gobierno nacional ha creado por intermedio del Ministerio de Salud y Protección Social, muchos de los cuales son garantías y mecanismos de protección contractual dirigidos a la adecuada prestación de un servicio esencial por propia definición legal (ley 100/1993, art. 4, inc. 2). En el mencionado sistema se excluyen autoridades, proveedores y usuarios cobijados por los regímenes especiales de salud y que, de manera reservada, se ocupan de desarrollar sus propias regulaciones para la atención en salud de sus usuarios cualificados.

Hecha la anterior salvedad, la ley 100/1993 como estatuto general del sistema de atención en salud, es el pilar en el cual se fundamentan las regulaciones y políticas del Gobierno nacio- 
nal en materia de garantía, calidad, auditoría e información pública de los servicios de salud. De acuerdo con ella, este servicio es una función pública cardinal que debe orientarse con los principios rectores establecidos como la accesibilidad, oportunidad, seguridad, idoneidad profesional, atención humanizada, satisfacción del usuario y estándares aceptados, que, entre otros, son de carácter obligatorio para el Estado y para los promotores y prestadores del referido servicio ya sean públicos o privados.

La política nacional de prestación de servicios como sistema de direccionamiento, tiene el propósito de garantizar el acceso, mejorar la calidad y generar eficacia en el uso de los recursos destinados para cada servicio y, en materia de atención en salud, esta política nacional se manifiesta a través de las entidades que integran el SGSSS, entre ellas, el Ministerio de Salud y Protección Social, el Consejo Nacional de Seguridad Social en Salud, la Comisión de Regulación en Salud, las entidades promotoras de salud (EPS) del régimen contributivo y del régimen subsidiado, el Fondo de Solidaridad y Garantía (Fosyga), las instituciones prestadoras de servicios de salud (IPS), el Instituto Nacional de Salud, el Instituto Nacional de Vigilancia de Medicamentos y Alimentos y las entidades territoriales de salud (Supersalud, 2007).

En virtud de la anterior política, surgió el SOGCS, como el conjunto de mecanismos, requisitos y procesos cobijados por la normatividad que define los parámetros mínimos de calidad, seguridad y garantía que deben ofrecerse en la prestación de servicios médico-asistenciales. También fomenta la competencia entre las EPS y entre las IPS, según un ideal de favorecimiento de los usuarios y, establece un sistema de información al público; todo con el objetivo particular de generar, mantener y mejorar la calidad y proteger de este modo a los destinatarios del servicio de manera integral (Ministerio de Salud y Protección Social, 2006).

El SOGCS ejerce su fuerte influencia sobre la prestación del servicio de salud guiándose con los principios registrados en el decreto 1011/2006, los cuales se complementan entre sí, estos son: la accesibilidad, entendida como posibilidad material y efectiva del usuario de utilizar los servicios que garantiza el sistema; la oportunidad, referida a la disponibilidad de los mismos cuando y donde se precisan; la seguridad como la estructura, métodos e instrumentos científicos o técnicos que aplicados de forma idónea garanticen un resultado cuando a ello haya lugar, o que de manera general y mayoritaria soporten un servicio adecuado y diligente que mitigue la posibilidad de consecuencias adversas en la ejecución de aquellos procedimientos que contienen obligaciones de medios a cargo del proveedor del mismo. La pertinencia, que resulta de vital importancia al evaluar el procedimiento menos lesivo para la salud del paciente y que al tiempo redunde en beneficio del usuario según sus necesidades y, finalmente la continuidad, que es la secuencia lógica y no interrumpida del tratamiento aplicado al usuario sea en el tiempo $\mathrm{o}$, en su intensidad y modalidad hasta tanto sea necesario o recomendado por las normas de la medicina.

\section{Componentes del Sistema Obligatorio de Garantía de Calidad en Salud}

ElSOGCS exige aplicar los principios descritos en la ejecución del servicio, pero al mismo tiempo determina como obligación y requisito para poder prestarlo, estar capacitado para cumplir con los cuatro componentes básicos del sistema, que son equivalentes entre sí y que deben obedecerse de forma armonizada e inseparable, como máxima garantía de calidad en la prestación de la salud. Estos componentes los desarrolla de forma amplia la normatividad vigente, concretándose en el Sistema Único de Habilitación, el Sistema Único de Acreditación de las EPS e IPS, el Sistema Único de Auditoría y el Sistema Único de Información y de Atención a los Usuarios.

En consecuencia, los principios antes vistos y los componentes variados $e$ independientes entre sí, concuerdan en un fin concreto que es la protección del derecho del usuario con un carácter 
poliédrico e integral, mismo que se manifiesta en el acceso efectivo al sistema de salud, en la garantía de calidad en cada fase médicoasistencial y, en las posibles acciones judiciales o prejudiciales que puede llegar a adelantar un usuario para obtener la reparación o indemnización de un perjuicio, luego de verse afectado por las acciones $\mathrm{u}$ omisiones en cabeza de un promotor o prestador, que resulten contrarias a los principios del sistema.

Este propósito del sistema de salud, funciona similar al modelo del Estatuto del Consumidor (ley 1480/2011), pues en este último los principios que aparecen en su artículo primero y los derechos y obligaciones que se consignan en los artículos 3, 6, 7 y siguientes, actúan mancomunadamente con las medidas de reparación y garantía existentes, con el objetivo de hacer real y manifiesta la protección del derecho del consumidor final (Corte Constitucional, 2000), articulando las funciones generales en aras de vigilar y materializar el cumplimiento de los fines del Estatuto. La Corte Constitucional mediante sentencia C-1141/2000 se pronunció respecto del carácter poliédrico de los derechos del consumidor indicando que:

Su objeto, en efecto, incorpora pretensiones, intereses y situaciones de orden sustancial (calidad de bienes y servicios; información); de orden procesal (exigibilidad judicial de garantías; indemnización de perjuicios por productos defectuosos; acciones de clase etc.); de orden participativo (frente a la administración pública y a los órganos reguladores).

De acuerdo con la Corte Constitucional (2000) la protección del consumidor busca cubrir integralmente cada escenario previsto por la ley donde pueda encontrarse el consumidor o usuario y garantizar en todas las fases, un ejercicio pleno del derecho colectivo que reconoce la Constitución Política y ahora desarrolla el nuevo Estatuto del Consumidor. Tal como se verá a continuación, a pesar de la coincidencia en el funcionamiento, estructura y orientación de ambos sistemas, los principios y componentes que trae consigo el SO-
GCS brindan un escenario de garantía y protección mucho más amplio y generoso que el propio de la ley 1480/2011 tal, pues son piezas primordiales para asegurar la prestación del servicio de salud y el correcto funcionamiento del sistema.

\section{Componente de habilitación}

El Sistema Único de Habilitación en salud maneja los estándares básicos de calidad que deben ofrecer los prestadores o proveedores del servicio y que son imprescindibles para garantizar seguridad a los usuarios; se trata entonces de una función eminentemente preventiva, que mitiga y reduce a su mínima expresión los potenciales riesgos que surgirían de la prestación del mismo por parte de entidades no capacitadas, cuya praxis podría comprometer seriamente el acceso y debida diligencia en su ejecución.

Este primer componente expuesto con holgura en la resolución 1441/2013 del Ministerio de Salud y Protección Social, tiene como naturaleza excluir a las IPS que carecen de la capacidad tecnológica, científica, administrativa y financiera, o que no alcancen al cumplimiento cabal del manual obligatorio de habilitación que acompaña con la misma fuerza normativa a la resolución 1441/2013, no pudiendo prestar servicios de salud en caso de no alcanzar ciertos mínimos exigidos.

Además ordena para las IPS, para las unidades móviles de salud -ambulancias y similares- $e$ incluso, para los médicos particulares -excluidos en el ejercicio de su profesión liberal de ser considerados parte en las relaciones de consumo analizadas en el sector de la salud- la necesidad de obtener el permiso de habilitación, que debe renovarse cada año a partir del 1 de junio de 2014 , una vez venzan los cuatro años de vigencia del primer permiso (resolución 1441/2013, art. 5).

\section{Componente de auditoría}

El Sistema Único de Auditoría como segundo componente, se encarga de evaluar el cumplimiento de los estándares de calidad complementarios a los que se fijan como básicos por el Sistema 
Único de Habilitación, a la vez que se ocupa de prever, advertir e informar de los problemas que puedan llegar a convertirse en obstáculos para el éxito de los procesos y la obtención de resultados esperados dentro de la gestión de un proveedor determinado del servicio. De acuerdo con el artículo 2 del decreto 1011/2006 "Es el mecanismo sistemático y continuo de evaluación y mejoramiento de la calidad observada respecto de la calidad esperada de la atención de salud que reciben los usuarios".

El Ministerio de Salud y Protección Social (2007) afirma frente a este componente que la función de auditoría para el mejoramiento de la calidad del servicio y la atención en salud, solo puede cumplirse por cada organización promotora o proveedora del servicio, si se realizan las siguientes actividades:

- Identificación de problemas de calidad (procesos prioritarios) con base en el seguimiento a riesgos del sistema único de habilitación, la autoevaluación realizada frente a los estándares de acreditación, los indicadores y los eventos adversos trazadores del sistema de información para la calidad.

- Establecimiento de la meta respectiva: definición de la calidad esperada.

- Medición y seguimiento del desempeño de los procesos prioritarios: identificación de la calidad observada.

- Aplicación y seguimiento de acciones de mejoramiento consistentes, tendientes a disminuir la brecha entre la calidad esperada y la calidad observada.

Este componente se sirve del análisis de resultados institucionales para verificar y promover el alcance de las metas y las expectativas de calidad propuestas, a la vez que progresivamente se reflejen en un servicio de mayor calidad y compromiso con los usuarios; asimismo cuestiona y censura toda situación que pueda generar detrimento o riesgo a la salud de los usuarios, canalizando su corrección, en armonía con los demás componentes del sistema. En síntesis, la auditoría vigila y constata el óptimo desempeño del servidor en aplicación de los principios de eficiencia pública, centralizando la información referente al servicio y sus condiciones reales y esperadas de prestación, para valorar el desempeño y establecer las medidas tendientes al fortalecimiento de la calidad en servicio y función del usuario de salud.

\section{Componente de acreditación}

El Sistema Único de Acreditación de las EPS, IPS, entidades de medicina prepagada y entidades territoriales de salud, se presenta como componente y criterio de calificación que busca incentivar el mejoramiento y la buena práctica de la medicina institucional, al tiempo que reconoce la importancia que la ley 100/1993 le otorgó a la libre competencia empresarial en el sector de la salud (Jaramillo, 1997). En este sentido, un sistema donde sus proveedores y prestadores se encuentran en constante búsqueda de crecimiento institucional y resultados que acrediten su calidad ante la competencia, tiene buenas razones para producir efectos favorables en el servicio que se ofrece y por lógica debería mejorar la atención, la cobertura y probablemente los costos del mismo. Según el propio sistema,

La acreditación en salud es una metodología de evaluación externa, establecida y reconocida desde hace casi 50 años en Norteamérica, específicamente diseñada para el sector salud, [...] que permite asegurar la entrega de servicios de salud seguros y de alta calidad. Se basa en requisitos de procesos de atención en salud, orientados a resultados [...] de las entidades que desean acreditarse y de reconocimiento público en el ámbito nacional e internacional, con efectos de mejoramiento de calidad demostrados. Esta evaluación corresponde a un proceso voluntario en el cual se reconoce públicamente [...] el cumplimiento de estándares superiores de calidad [...] 


\section{Componente de información y atención}

El último componente es el Sistema de Información en Salud y Atención a los Usuarios, que agrupa dos esferas prácticas con alcances y objetivos diferentes entre sí; el primer enfoque funciona como un banco de datos nacional, que opera como canal por el cual fluye la información relevante de la salud. De este sistema se sirve el Gobierno nacional, las entidades territoriales, las EPS e IPS, los centros de investigación y las universidades, quienes lo utilizan para estandarizar la información existente y descentralizar su acceso hacia todos los operadores regionales y locales.

El sistema busca que la información de envergadura sea difundida y esté al alcance real de cada prestador, quien puede tener acceso a la información y recibir conocimiento actualizado en materia de tratamientos médicos, contingencias, nueva normatividad de la disciplina, alertas tempranas para la suspensión del uso de medicamentos o tratamientos, entre muchos otros datos de gran valor para la prestación adecuada y debidamente informada del servicio (Presidencia de la República, 2012).

La segunda esfera o enfoque práctico de este sistema ya no se dirige al servicio, sino al paciente, su objetivo es construir medios idóneos para generar el conocimiento integral del que precisa el usuario en sede asistencial o quirúrgica, de tal modo que aquel pueda otorgar el consentimiento informado para acceder al procedimiento o, en su lugar, sean sus familiares con representación legal o circunstancial quienes lo concedan cuando se trate de un paciente incapaz de expresar su voluntad por razones legales o médicas.

Además del consentimiento informado, el sistema coloca a disposición del usuario, toda la información complementaria del servicio, de suerte que la persona que acuda en cualquier otra fase de su prestación, sea administrativa o básica, pueda recibir en detalle y a satisfacción una orientación en la tramitología propia de cada EPS o IPS, así como los conductos regulares para presentar solicitudes, quejas o reclamos, armonizando las funciones de este componente con las garantías y mecanismos que puede ejercer el usuario para hacer valer sus derechos cuando considere que no están siendo respetados.

\section{Aproximación del Sistema Obligatorio de Garantíade Calidad en Salud al Estatuto del Consumidor}

Al adentrarse un poco más en el Estatuto del Consumidor, es posible apreciar que sus artículos 6 y 7 , colocan a cargo de los productores y proveedores de bienes y servicios ciertas responsabilidades y deberes de obligatorio cumplimiento frente al consumidor o usuario, incluida la denominada garantía legal. Sin embargo, al momento de considerar la atención en salud, resulta muy complicado adaptar y aplicar dichos conceptos claves del Estatuto a situaciones comúnmente presentadas en el sistema de salud, porque al tratarse de un servicio profesional, de alta complejidad, es necesario acudir a las amplias regulaciones que de forma especial acompañan el SOGCS y que, solo por su conducto permiten determinar por ejemplo, cuándo se ha incumplido con la garantía legal regulada paralelamente por el Estatuto, en los casos de obligaciones de medio adquiridas con los pacientes y usuarios. Que por lo general es cuando se desconocen las condiciones de calidad en la prestación del servicio, según las condiciones establecidas en normas de carácter obligatorio, en las ofrecidas o en las ordinarias habituales del mercado (artículo 7), surgidas mayoritariamente en los tratamientos y procedimientos médicos de gran complejidad.

Ahora bien, al hacer una aproximación de estos componentes al Estatuto del Consumidor, vienen a relucir en este las disposiciones de seguridad, garantía legal y calidad en los bienes y servicios ofrecidos al público, así como su objetivo de preservación y efectividad de los derechos de los consumidores y usuarios (Congreso de la República, 1993). Con todo, no se evidencia en el Estatuto la implementación obligatoria de componentes como el Sistema de Habilitación o de Auditoría del SOGCS, siendo criterios que 
resultarían muy valiosos para la estandarización de cada proceso productivo en el mercado general de bienes y servicios, pues si bien es cierto, las autoridades de control supervisan en cierta medida (Gual y Villalba, 2013) el ejercicio profesional de los comerciantes y partícipes de la cadena de distribución, es también verdad que no se plantea un deber ser que tenga la vocación de excluir anticipada y preventivamente a un prestador de un servicio ${ }^{1}$ o productor de bienes tradicionales que no cuente con ciertas condiciones y garantías de calidad para los usuarios y consumidores. La vigilancia que se realiza hoy en día es posterior, pero descuida en la mayoría del mercado las labores de prevención que podrían efectuarse de manera anticipada con resultados posiblemente muy favorables.

Es decir, aun cuando el decreto 3257/2008 expresa con claridad que el Subsistema Nacional de Calidad promueve en los mercados la seguridad, la calidad, la confianza, la productividad y la competitividad de los sectores productivo $e$ importador de bienes y servicios, y protege los intereses de los consumidores en los asuntos relativos a procesos, productos y personas; y por otro lado, coordina las actividades que ponen en marcha las instancias públicas y privadas relacionadas con la formulación, ejecución y seguimiento de las políticas sobre normalización técnica, elaboración y expedición de reglamentos técnicos, acreditación, designación, evaluación de la conformidad y metrología; este no resulta adecuado frente a la prestación del servicio de salud.

Resulta entonces preciso indicar que la normatividad en salud está permanentemente enriquecida por la infinidad de reglas de conducta y procedimientos asistenciales y por manuales que

1 Se trata de "servicios" porque en productos el subsistema general de calidad cumple un rol importante. El control general referenciado no resulta aceptable ni factible, porque afecta al comercio y no es viable económicamente verificar previamente la calidad de todos los productos. En cuanto a "servicios" menos, porque la calidad depende de la forma en que se prestó el servicio y esto solo se sabe con la prestación efectiva del mismo caso a caso. guían el ejercicio profesional en el uso habitual o restringido de medicamentos, la aplicación preventiva de tratamientos, el diagnóstico y cirugía, entre otros que, en conjunto determinan el deber objetivo de cuidado como reglas de obligatorio cumplimiento para sus prestadores en todas las fases del servicio. Por ende, determinar si se ha cumplido o no de manera diligente con un procedimiento, resulta mucho más complejo aun cuando no imposible, que detectar la falla de un servicio tradicional como podría ser la telefonía o la televisión por cable, en donde basta la demostración fáctica y simple de la ausencia de tono en la línea o en la transmisión televisiva, para obtener su reparación o reclamarla ante la autoridad para hacer efectiva la garantía.

\section{El régimen de responsabilidad con el usuario}

En cuanto a responsabilidad y garantía del servicio de salud, establecer si se ha incumplido con el deber de cuidado y diligencia es tan subjetivo como el mismo régimen de culpa probada del servicio en materia de reparación directa-artículo 90 de la Carta Magna- o como la determinación de la tipicidad y la culpabilidad de una conducta en materia penal, siendo esto, algo que le distancia mucho del régimen objetivo del Estatuto del Consumidor. Cuando se debe estudiar, conocer y finalmente juzgar la conducta de un profesional o proveedor de salud al momento de ejecutar un servicio relacionado con esta, lo que debe hacerse es conocer las conductas señaladas por los reglamentos o por los estatutos y manuales públicos que enseñan el debido ejercicio de la salud.

Es decir, en caso de que una de las directrices que son ordenadas por este compendio no sea acatada o haya sido ignorada, necesariamente implica que se ha vulnerado el deber objetivo de cuidado con una conducta negligente y por lo mismo, si genera afectación al derecho material del usuario se estará incumpliendo con las normas que aseguran la calidad e idoneidad del servicio, dando lugar a la determinación de responsabilidad contractual del prestador. 
En la misma línea de argumentación, puede afirmarse que el Estatuto del Consumidor tiene grandes virtudes al definir el concepto de producto defectuoso, sin embargo, deja vacíos al momento de precisar o al menos considerar el significado de un servicio defectuoso (Fernández, 2013). Si bien es cierto hay algunas circunstancias propias de la regulación del consumidor que se reservan a los bienes, al definir estos conceptos tan claves en la determinación de responsabilidad del proveedor como el defecto de un elemento que se pone a disposición del mercado y con destino al consumidor, es menester cubrir este tipo de vacíos legales, porque los servicios sin lugar a dudas ocupan hoy en día un lugar muy significativo al hablar de volumen de mercado, incluso más todavía que los mismos bienes, y al intentar subsumir el concepto "defectuoso" en cualquier servicio y en particular en la atención en salud, resulta igualmente insuficiente desde la perspectiva de la ley 1480/2011.

A manera de ejemplo se propone un caso de la atención médico-asistencial integrado por obligaciones de medios: una persona que padece cierta enfermedad recibe un tratamiento destinado por el proveedor de salud para su atención, siendo distinto al que su enfermedad requiere, puede ser por desconocimiento o por error de diagnóstico al no identificar la patología realmente padecida, dando como resultado, la muerte o el perjuicio físico del usuario, todo a partir de un servicio defectuoso que se inició en un diagnóstico médico equivocado.

Si bien puede decirse que el tratamiento fue correcto o diligentemente aplicado $\mathrm{y}$, aunque no se exige un resultado favorable, es aún más cierto que el servicio fue defectuoso por un mal diagnóstico que incumplió con uno de los principios que contiene el SOGCS como lo es la pertinencia y, además, ignoró los reglamentos y manuales preventivos y de seguridad que deben seguirse en la prestación del servicio.

En un caso así, la discusión sobre la responsabilidad y su procedencia desde el punto de vista del Estatuto del Consumidor indicaría que, para poder precisar si hay lugar a ella, debe estar presente una falla verificable de la garantía legal, o una falla del servicio con una demostración típicamente objetiva para su solución inmediata; pero en los servicios de salud, donde por regla general, las obligaciones a cargo del proveedor son de medios (Fernández, 2013), la demostración de la diligencia o su ausencia es determinante, siendo el régimen subjetivo y probado el que corresponde aplicar, teniendo que acudir necesariamente a los mecanismos tradicionales como la acción de tutela o en ocasiones el desvalorizado derecho de petición que ofrece el sistema de salud al usuario, por resultar mucho más adecuado al momento de satisfacer y reparar los intereses posiblemente afectados.

Cabe anotar que este aspecto se interpreta en el entendido que la carga de la prueba la tiene el profesional y no el consumidor según el Estatuto, esto es, la ley 1480/2011 establece causales de exoneración tanto para garantía como para responsabilidad por servicios defectuosos y en ninguna aparece la prudencia y diligencia; circunstancia que puede ser más útil para el consumidor a la hora de reclamar por las soluciones que desee.

Además, teniendo en cuenta que en el plano médico-asistencial resulta de gran dificultad señalar con celeridad el defecto en el servicio, al tratarse de un entorno potenciado con tratamientos complejos, algunas veces de larga duración que no permiten demostrar tan fácil y rápidamente la falla, como para proponer la aplicación de una medida como la garantía inmediata que ofrece el régimen general condensado en el Estatuto; inmediatez que dicho sea de paso, se trata de un ápice que le da su naturaleza a la garantía legal, caracterizándola por la procura de la pronta satisfacción de los derechos del consumidor. Característica que, conforme a lo expuesto, no es extensible a la garantía que pueda exigirse en relación con un defecto presentado en la prestación del servicio de salud.

En todo caso, no se descarta para futuras discusiones la posibilidad de revisar y estudiar su 
aplicabilidad en materia de salud a procedimientos puramente administrativos o de menor complejidad.

Como se anotó en el ejemplo dado, al tratarse de defectos del servicio existe una problemática que impide la aplicación del Estatuto del Consumidor, misma que se extiende a las opciones ofrecidas al consumidor en su artículo 11 numeral 3, tales como "La prestación del servicio en las condiciones en que fue contratado" o la "devolución del precio pagado", puesto que resultan insuficientes por sí solas para responder interrogantes como ¿de qué manera restablece esta sanción legal el derecho del usuario que ha fallecido o que ha sufrido graves e irreversibles lesiones en su salud o condición personal? Pensándose en que para lograr dicho restablecimiento a este en igualdad de condiciones con los consumidores de bienes, le baste emplear tan solo las herramientas que brinda el mismo Estatuto, sin que necesariamente tenga que acudir a la justicia ordinaria para solicitar el reconocimiento y pago de perjuicios en "garantía" como hasta ahora tendría que hacerse.

¿Cómo podría efectivizarse la garantía legal con la devolución del dinero en un sistema con aportes mensuales del régimen contributivo? $\mathrm{O}$ peor aún, en el régimen subsidiado, aclarándose sobre el particular, que lo dicho aplica a todos los regímenes, inclusive sistemas complementarios o medicina prepagada, considerando que el servicio de salud debe prestarse siempre sin que la pertenencia a uno $\mathrm{u}$ otro régimen o a uno $\mathrm{u}$ otro tipo de programa o modalidad de prestación, deba interferir en dicho goce o disfrute.

Lo anterior facilita concluir que el tratamiento de la responsabilidad del productor y proveedor plasmado por el Estatuto del Consumidor, dista claramente del sistema de atención en salud en aspectos tan sustanciales como la clase de responsabilidad a aplicar, donde sea del caso advertir, es el régimen subjetivo en el estudio de la conducta y la determinación de la culpa (Jaramillo, 2010) el que desempeña un papel en verdad importante en la evaluación de responsabilidad dentro de un servicio humano y personalizado que sigue normas a veces muy específicas y de difícil interpretación.

Según esto, la prestación de un servicio que por su naturaleza esencial resulta obligatorio, a veces no tiene claridad sobre temas como la libertad y autonomía contractual o la libre competencia sin restricciones más allá de la lealtad y la legalidad, siendo natural que algunos no sean aplicables como mecanismos de protección contractual en beneficio del usuario de los servicios de salud, reafirmando de nuevo que las medidas tradicionales de defensa, garantía y seguridad propias del SOGCS resultan aplicables por prelación y por relevancia.

\section{Mecanismos de reclamación del usuario y responsabilidad}

La atención en salud parte del principio de su accesibilidad universal, es decir, que toda persona pueda, en cualquier momento que lo necesite, tener asegurado el ingreso al sistema y así obtener tratamiento conforme a sus necesidades. El régimen de salud comprende entonces que la primera problemática que afecta la efectividad del servicio o derecho, es la posibilidad real de acceder a él; y es justo aquí donde se puede encontrar el primer mecanismo dispuesto para proteger al usuario, siendo el más directo y obvio de todos, a saber: una atención personalizada.

Bien se ha dicho a lo largo del presente documento que la salud puede haberse transformado en su operatividad y haber evolucionado a su institucionalización, pero los principios que hoy la rigen, demuestran por lo menos en teoría, que sigue teniendo el carácter humanizado que siempre debe conservar un servicio tan cercano a la esencia misma de la persona; por ello las entidades y autoridades que aseguran, promueven y prestan la salud, deben colocar a disposición de todos los usuarios del sistema las herramientas que faciliten recibir de manera eficaz y oportuna la atención que se precisa, en vez de crear barreras o limitaciones administrativas para acceder a los mencionados servicios (Supersalud, 2007). 
La orientación del usuario, por ejemplo, resulta ser relevante si se brinda de forma sencilla y comprensible, con un lenguaje adecuado que explique con claridad los procesos y trámites que deben adelantar las personas para acceder a lo que su salud requiera. Esta función corresponde a la materialización inicial del Sistema de Información al Usuario, particularmente en el segundo enfoque presentado ut supra, además de que es impuesto por el sistema de tal forma que, las entidades responsables deben disponer de un área que se ocupe de resolver las inquietudes y ayudar al usuario a efectuar las gestiones administrativas para obtener la atención en salud de acuerdo con la Superintendencia Nacional de Salud (2007).

También necesaria es la divulgación constante de información relacionada con los departamentos o secciones burocráticas responsables de cada fase de la atención del usuario, incluidos los datos de contacto y ubicación, con particular énfasis en las dependencias encargadas de la asignación de autorizaciones o citas médicas, así como la indicación de las IPS que forman parte de la red ofrecida por una EPS con su respectiva ubicación y mecanismos de acceso.

Siguiendo el conducto regular, los usuarios tienen como segunda instancia la oportunidad de acudir ante las IPS y ante las mismas autoridades integrantes del SGSSS para elevar solicitudes, quejas o reclamos vinculados con la atención recibida; para lo cual el sistema fomenta la creación de espacios de participación ciudadana que sean ofrecidos con el patrocinio de la tecnología y la comunicación, de tal manera que los aportes ciudadanos sean considerados y puedan llegar a significar un mejoramiento de calidad en la atención de salud (Presidencia de la República, 2012).

Además, la Superintendencia Nacional de Salud (2007) ha puesto a disposición de los usuarios el "formato de negación de servicios" que según la misma funciona como un:

[...] instrumento a través del cual, cuando le sea negado un medicamento o un servicio, el afectado tendrá derecho a que se le dé una constancia por escrito de las razones por las cuales no le han sido proporcionados los medicamentos, procedimientos o intervenciones solicitados a la entidad aseguradora o prestadora y determinar si podía acceder a los mismos de acuerdo con la cobertura del plan de salud que le está siendo prestado por la entidad aseguradora.

Este formato no tiene límites en el sistema de salud, incluso trasciende a los regímenes especiales de salud como el de las Fuerzas Militares y otros tantos más, comprendiéndose como el derecho que toda persona tiene de exigir que, frente a la negación del servicio o del suministro de un medicamento, se haga constar por escrito la información que sustenta la conducta de la EPS o IPS, quien tiene la obligación de diligenciarlo y certificarlo.

Cuando se trata de la negativa de suministrar un medicamento, la instancia que puede seguirse inmediatamente al formato de negación, es dispuesta por el sistema a través del Comité Técnico Científico, quien tiene la autoridad de definir si el medicamento se debe proporcionar o no al usuario. Son comunes los casos asociados al suministro de medicamentos que están por fuera del plan obligatorio de salud, donde el Comité Técnico Científico ha determinado en segunda instancia la obligación de suministrarlos en cabeza de las EPS, luego de la evaluación concreta de las circunstancias personales del usuario y la enfermedad que padece; siendo usual que los pacientes de cáncer, sida y otros padecimientos de alto costo sean beneficiados por este tipo de protección especial que les concede el sistema.

Es además importante resaltar que, la acción de tutela ha tenido un protagonismo muy frecuente al momento de ordenar el mismo suministro de estos medicamentos, cuando las EPS no han tramitado la consulta ante el Comité en mención después de haber tenido la obligación de hacerlo, o cuando han hecho caso omiso de sus órdenes, pues en estos casos, el derecho a la salud adquiere conexidad vital con el núcleo sólido de derechos fundamentales. 
Cuando persista la inconformidad del usuario a pesar de haber ejercido los anteriores mecanismos de protección, este puede acudir a la Superintendencia Nacional de Salud quien se encarga de la inspección, vigilancia y control de la prestación de servicio, tratándose de un organismo con facultades sancionatorias que tiene como función principal, encaminar sus esfuerzos en la supervisión del SGSSS y vigilar que se respeten los derechos de los usuarios.

El decreto 1018/2007, las leyes 1122/2007 y 1438/2011 y la resolución 3140/2011 entre otras fuentes, sustentan esta facultad y establecen que la Superintendencia Nacional de Salud y sus delegadas pueden emprender procedimientos administrativos sancionatorios en contra de sus vigilados, entre ellos EPS e IPS, el mismo Fosyga y otros partícipes del servicio de salud determinados en el artículo 121 de la ley 1438/2011, cuando su conducta atente contra el SGSSS o contra los derechos y garantías de los usuarios, facultando incluso al superintendente Nacional de Salud, para decretar medidas cautelares inmediatas de conformidad con el artículo 125 ibidem, para ordenar "la cesación provisional de las acciones que pongan en riesgo la vida o la integridad física de los pacientes o el destino de los recursos del Sistema General de Seguridad Social en Salud" así como para que "se adopten las correspondientes medidas correctivas o de saneamiento" (decreto 1018/2007, art. 8).

Dentro de las facultades sancionatorias que se otorgan a la Superintendencia y que desarrolla la resolución 3140/2011, resulta primordial resaltar una de las disposiciones más significativas en materia de garantía y protección extensiva de los derechos de los usuarios de la salud y en particular de quienes padecen enfermedades de alto costo como el cáncer, se trata de la ley 1384/2010 también conocida como "Ley Sandra Ceballos", a partir de la cual se estructuró un régimen sancionatorio en contra de las entidades prestadoras de salud que desconozcan el derecho de atención integral de la población afectada con este tipo de enfermedad, dando como resultado que sea posible la imposición de multas entre los 200 y los 1000 SMLMV y la cancelación de licencias de funcionamiento, en los escenarios ya conocidos de negación de tratamientos y medicamentos, o la oposición a las decisiones del Comité Técnico Científico.

Finalmente, el último recurso al que puede acudir el usuario, además de la acción de tutela, es como ya se anticipó, la justicia ordinaria, como vía para adelantar las respectivas reclamaciones que por responsabilidad médica contractual puedan configurarse en la prestación negligente o defectuosa del servicio, así como en la insatisfacción del resultado esperado en áreas como la obstetricia y procedimientos de cirugía estética (Jaramillo, 2011).

Para estos efectos es interesante considerar que, dentro de las citadas medidas jurisdiccionales, existe un ambiente favorable que ha reconocido en los últimos años las condiciones desventajosas que enfrentan los usuarios de salud ante el proveedor de los servicios. Por ejemplo, la Corte Suprema de Justicia (Fernández, 2013) y en algún momento el Consejo de Estado, decretaron la aplicación de un régimen probatorio comprensivo con la capacidad probatoria real que está en cabeza de un paciente del común, quien no entiende muchas veces su propia historia clínica ni mucho menos las condiciones de ejecución de un tratamiento; surgiendo la aplicación de un régimen probatorio invertido, en el cual el demandante que pretende el resarcimiento de un perjuicio, no debe asumir la carga de probarlo como históricamente el artículo 177 del Código de Procedimiento Civil (CPC) ordenaba, toda vez que el verdaderamente capacitado para hacerlo es su contraparte, quien con especialidad y profesionalismo conoce y dispone de todos los medios para probar si fue o no diligente en el cumplimiento de su deber objetivo de cuidado al prestar el servicio médico-asistencial, con lo que se reitera el carácter dinámico de la carga de la prueba, que al menos en materia de consumo, siempre ha existido, a diferencia como se señaló, de lo dispuesto en el CPC.

Sin embargo, es igualmente importante destacar que este régimen ha tenido cierta inseguridad 
e indeterminación jurídica en los últimos años, por cambios dogmáticos del Consejo de Estado y por la implementación de un régimen de la carga dinámica de la prueba, con el que ya no se tiene por regla general que el usuario se vea beneficiado de una presunción legal a su favor, en la que se considere su condición de inferioridad con relación al prestador del servicio; sino que el juez decidirá en cada caso particular si invierte la carga probatoria al demandado o la sostiene en el demandante.

\section{Conclusiones}

- La visión y desarrollo económico de la salud, así como su enfoque empresarial e institucional y los fundamentos prácticos que guían su prestación, transformaron el vínculo tradicional del médico-paciente en lo que hoy podemos definir como una relación de consumo de servicios de salud. No obstante, pese a su relación directa con el Estatuto del Consumidor, este pierde en muchos aspectos la vocación para regular aquellas relaciones especiales de consumo, principalmente porque sus medidas de garantía, protección y satisfacción no están desarrolladas con tal suficiencia, como para alcanzar el nivel exigido por los servicios médico-asistenciales que reciben a diario los miles de usuarios del sistema general de atención en salud, los cuales además, tienen respaldo constitucional, mismo que ha flexibilizado todos los campos del derecho en los que antes imperaban únicamente principios como la autonomía de la voluntad privada, que hoy cede ante la prevalencia de protección efectiva de derechos constitucionales como el de la salud, que adquiere el carácter de fundamental por la conexidad que el mismo aguarda con el derecho a la vida, debiendo aludirse entonces a una complementariedad entre los dos sistemas.

- La atención en salud del usuario, al menos en los ámbitos teórico y normativo, está robustecida por los principios rectores de la función pública y además, forma parte de los fines esenciales del Estado como un derecho y servicio obligatorio, que como tal torna irrenunciables los derechos y garantías reconocidos para el usuario y que recibe las directrices de la política nacional de servicios, por lo que sus garantías en materia de calidad del servicio, protección, información y acceso oportuno cuentan con un campo mucho más amplio y preferente que se superpone al Estatuto del Consumidor. Lo que evidencia que en efecto, existe un régimen más garantista de los derechos que como consumidor tiene quien es usuario de los servicios de salud, a quien a falta de regulación específica en el Estatuto del Consumidor, por vía de otras alternativas, como la existencia de un régimen especial de aplicación preferente (que resulta más acorde con las necesidades y el contexto de los pacientes-consumidores) se le han salvaguardado todas sus facultades, en comparación con la regulación ofrecida para los consumidores de otro tipo de servicios.

- Y es que justamente con ocasión de la aplicación de dicho régimen especial dispuesto para el sector salud, mismo que condensa las herramientas antes expuestas en favor de los consumidores del servicio; se hace palpable el carácter garantista previsto normativamente para el sector. No obstante ello, debe contrastarse con su aplicación práctica, que de acuerdo con lo difundido en medios masivos de comunicación, no resulta ser la más satisfactoria con todo y el andamiaje normativo antes señalado.

- Componentes del SOGCS tales como el Sistema Único de Habilitación, debieran ser inherentes al universo de las relaciones de consumo, en este sentido, considerarlos en el futuro dentro de la protección usual del consumidor, puede ofrecer crecimiento en materia de garantías preventivas a favor del resto de los consumidores y usuarios de productos y servicios tradicionales; ampliando así el marco general de protección a los consumidores, brindando una protección integral para los mismos. 


\section{Referencias}

Fernández, M. (2013). La transposición del modelo de consumo al campo médicosanitario. En: J. Gual \& J. Villalba (dir). Derecho del consumo, problemáticas actuales. Bogotá: Grupo Editorial Ibáñez.

Gual, J. \& Villalba, J (Dir.) (2013). Derecho del consumo, problemáticas actuales. Bogotá: Grupo Editorial Ibáñez.

Guzmán, F. \& Redondo, H. (1998). La ley 100 de 1993: ¿a quién beneficia realmente? Revista Universidad del Rosario, 91(581), 45.

Jaramillo, C. (2010). La culpa y la carga de la prueba en el campo de la responsabilidad médica. Bogotá: Grupo Editorial Ibáñez.

Jaramillo, C. (2011). Responsabilidad civil médica "la relación médico-paciente" análisis doctrinal y jurisprudencial. Bogotá: Grupo Editorial Ibáñez.

Jaramillo, I. (1997). Libre competencia en la prestación de la salud. "El futuro de la salud en Colombia. La puesta en marcha de la ley 100”. Bogotá: Fundación Corona.

Ministerio de Salud y Protección Social. (2007). Sistema Obligatorio de Garantía de Calidad en Salud. Pautas para el mejoramiento de la calidad de la atención en salud. Bogotá: Ministerio de Salud y Protección Social.

Presidencia de la República. (2012). Urna de cristal. ¿Sabías que entró en marcha el Sistema Único de Información en Salud de Colombia? Disponible en: http://www. urnadecristal.gov.co/gestion-gobierno/sabas-que-entr-en-marcha-sistema-nico-deinformaci-n-en-salud-de-colombia

Superintendencia Nacional de Salud. (2007). Guía práctica "Así protegemos los derechos de los usuarios de la salud". Bogotá: Superintendencia Nacional de Salud.

Villalba, J. (2012). Introducción al derecho del consumo. Bogotá: Universidad Militar Nueva Granada.

\section{Normatividad y jurisprudencia:}

Congreso de la República de Colombia. (1991). Constitución política.

Congreso de la República de Colombia. (1993). Ley 100 "Por la cual se crea el sistema de seguridad social integral y se dictan otras disposiciones".

Congreso de la República de Colombia. (2007). Ley 1122 "Por la cual se hacen algunas modificaciones en el Sistema General de Seguridad Social en Salud y se dictan otras disposiciones".

Congreso de la República de Colombia. (2011). Ley 1438 "Por medio de la cual se reforma el Sistema General de Seguridad Social en Salud y se dictan otras disposiciones".

Congreso de la República de Colombia. (2011). Ley 1480 "Por la cual se expide el Estatuto del Consumidor y se dictan otras disposiciones".

Consejo de Estado. (2005). Sentencia en acción popular 00254 de 10 de febrero. C. P.: María Elena Giraldo Gómez. Sobre la situación de desventaja del consumidor en la relación de consumo.

Consejo de Estado. (2006). Sentencia del 22 de febrero, expediente 2002-00540. C. P.: Rafael Ostau de Lafont Pianeta.

Corte Constitucional de Colombia. (1993). Sentencia SU-067. M. P.: Fabio Morón Díaz y Ciro Angarita Barón.

Corte Constitucional de Colombia. (2000). Sentencia C-1141. M. P.: Eduardo Cifuentes Muñoz.

Corte Constitucional de Colombia. (2002). sfefefN ${ }^{\circ} 10$.trato. el sponsabilidad ma leyabituales, en este sentido su consideracificar la fortSentencia C-377. M. P.: Clara Inés Vargas Hernández.

Corte Constitucional de Colombia. (2002). Sentencia C-973. M. P.: Álvaro Tafur Galvis. 
Corte Constitucional de Colombia. (2002). Sentencia C-1071. M. P.: Eduardo Montealegre Lynett.

Corte Constitucional de Colombia. (2003). Sentencia T-466. M. P.: Alfredo Beltrán Sierra.

Corte Constitucional de Colombia. (2012). Sentencia C-909. M. P.: Nilson Pinilla Pinilla.

Corte Constitucional de Colombia. (2014). Sentencia C-133. M. P.: Alberto Rojas Ríos.

Ministerio de Comercio, Industria y Turismo. (2008). Decreto 3257 "Por el cual se modifica parcialmente el Decreto 2269 de 1993 y se dictan otras disposiciones".
Ministerio de Salud y Protección Social. (2006). Decreto 1011 "Por el cual se establece el Sistema Obligatorio de Garantía de Calidad en Salud del Sistema General de Seguridad Social en Salud".

Ministerio de Salud y Protección Social. (2007). Decreto 1018 "Por el cual se modifica la estructura de la Superintendencia Nacional de Salud y se dictan otras disposiciones".

Ministerio de Salud y Protección Social. (2013). Resolución 1441 "Por la cual se definen los procedimientos y condiciones que deben cumplir los prestadores de servicios de salud para habilitar los servicios y se dictan otras disposiciones". 